\title{
Metaphors of Metaphors: Reflections on the Use of Conceptual Metaphor Theory in Premodern Chinese Texts
}

\section{Stefano Gandolfo ${ }^{1}$}

Published online: 16 July 2019

(C) The Author(s) 2019

\begin{abstract}
In this essay, I challenge the use of Conceptual Metaphor Theory (CMT) in the premodern Chinese setting. The dominant, implicit assumption in the literature is that conclusions reached by CMT on the ways in which cognition operates can be applied in toto and without qualification onto the makers of classical Chinese texts. I want to challenge this assumption and argue that textual evidence from premodern Chinese points to a different cognitive process. Differences in the use and conceptualization of image-based thinking as well as differences in the understanding of the relationship between words, images, and the world reveal a different cognitive process. However, I do not wish to argue for a total abandonment of the insights of CMT. Rather, I call for the need to properly qualify its findings in light of premodern Chinese use and conceptions of image-based language.
\end{abstract}

Keywords Conceptual metaphor · Image-based language $\cdot$ Classical Chinese

\section{Introduction}

Virtually all literature that applies Conceptual Metaphor Theory (CMT) on premodern Chinese texts does so uncritically. The dominant, implicit assumption is that the conclusions reached by CMT on the ways in which cognition operates can be applied in toto and without qualification onto the makers of classical Chinese texts. I want to challenge this assumption and argue that textual evidence from premodern Chinese points to a different cognitive process. Differences in the use and conceptualization of image-based thinking as well as differences in the understanding of the relationship between words, images, and the world indicate that a different cognitive process was

Stefano Gandolfo

stefano.gandolfo@stx.ox.ac.uk

1 St. Cross College, University of Oxford, 37A St. Giles st., Oxford OX1 3LC, UK 
taking place in the minds of the makers of premodern Chinese texts. Appreciating these differences brings us closer to the cognitive patterns of the communities of thinkers under study and reveals the cultural embeddedness of CMT, urging research in human cognition to reevaluate some of its conclusions on the universality of conceptual metaphors. However, I do not wish to argue for a total abandonment of the insights of CMT. Rather, I draw attention to the need to properly requalify its findings in light of premodern Chinese use and conceptions of "metaphor": image-based thinking happens in all linguistic communities but the mode behind the pattern differs due to cultural particularities. The current expressions of CMT should be seen as the manifestation of only one strand of image-based thought which has mistakenly been applied to all cultures without reflection or modification. Thus, the main goal of this essay is to reveal the presuppositions of CMT that make it culturally distinct. ${ }^{1}$

This essay is structured in three sections. In Section 2, I introduce CMT, its main assumptions and conclusions. I also present main variations to the theory, and reflections on the role of culture in CMT. I proceed with a brief overview of the literature that has applied CMT on premodern Chinese texts, paying special attention to argumentative strategies and linguistic expressions within the literature. I conclude Section 2 by introducing at greater length the key problem of this essay. In Section 3, I show the cultural specificity of CMT by examining the etymology, history, and theory of imagebased language in the Western and Chinese settings, showing the theoretical incoherence between CMT and the Chinese experience. In Section 4, I respond to specific points raised in the CMT literature on classical Chinese texts. I conclude with a brief discussion with suggestions for the need to modify rather than abandon the use of CMT, based on the proposition that CMT can illuminate the semantic creation of meaning more than it can reveal universal patterns of cognition.

This essay is defined by two methodological assumptions. The first is the idea that any theory on the cognitive role of image-based thinking will be based on the practice and reflection of image-based language. The use of "metaphors" and other linguistic tropes - metonymy, simile, and allegory or $f u$ 賦 (exposition), $b i$ 比 (comparison), and xing 興 (evocation), among others - informs the ways in which thinkers in different traditions and at different times have reasoned about the properties and functions of image-based language. Coupled with propositions about the role of language and its relationship to the world, reflection on linguistic tropes functions as the fundamental building block for contemporary cognitive theories of metaphor. Therefore, my main argument that the primary and secondary levels of image-based language - use of linguistic tropes and reflection upon them-found in the Chinese setting does not fully cohere with the conclusions of CMT rests upon this assumption. The second and larger assumption behind this essay and CMT as a whole is that the linguistic artifacts of a specific community provide meaningful access to the cognitive patterns of thought of the members of that community (Steen 2011: 28). In this essay, I accept this assumption (Cserep 2014: 265; Gibbs 2015: 169) since there are no means to directly access

\footnotetext{
${ }^{1}$ I do not examine the role of conceptual metaphors in modern Chinese because I take it that there is a clear and significant break between modern and premodern Chinese on the level of linguistic expression and cognitive configuration. Findings which suggest convergence in modern Chinese and English should not be used to argue for premodern Chinese ways of thought as the languages and contexts in which they are employed are significantly different. For the role of CMT in modern Chinese, see N. Yu 1998, Yang 2011, and Chang 2015.
} 
cognitive processes - especially of nonliving individuals and communities. Resorting to the linguistic, and particularly the written, artifacts of specific communities is a reasonable alternative route. Thus, all conclusions drawn in this paper are determined by these two fundamental methodological postulates.

My argumentation proceeds from a collection of texts from different periods. I am aware that macrogeneralized statements about "cultures" are often misleading, hiding more than they reveal (Ekstrom 2014). I ground my findings to specific texts as much as possible with the implicit understanding that these works are representative of a broader way of thinking because they are constitutive of that way of thinking. In other words, the selection of texts in this essay has been based on their relative influence over time, and while the makers of these texts were in conversation with each other, I do not take the texts as forming a clear-cut conceptual unit. Undertaking a proper historical review of the understanding of image-based language in the "Western" or "Chinese" setting is a monograph project on its own- I do not purport to have provided an exhaustive representation of different traditions but rather a plausibly dominant one while recognizing the great plurality within each. I hope this essay sparks further engagement with the issue of applying conceptual tools born out of the West to describe the West to radically different contexts - especially when such tools claim or prima facie appear to be culturally neutral.

\section{Laying the Background}

\subsection{Introducing CMT}

The 1980s saw a revolution in the study of metaphor. In Metaphors We Live By, Lakoff and Johnson put forth a set of propositions about metaphor which sparked great interest in the nature and function of metaphor in cognitive linguistics, philosophy, sociology, and beyond (Lakoff and Johnson 1980). Their key proposition is that metaphor is not an aspect of language but rather of thought: metaphors exist in a prelinguistic cognitive plane and structure in profound ways abstract ideas and ways of reasoning. A metaphor is understood as a mapping across different domains where the features of one, concrete, "source" domain are used to conceptualize an abstract, "target" domain. For example, phrases such as "She won the debate," "He defended his argument," "She attacked his position" reveal a conceptual structure of thinking about arguments as war. Lakoff and Johnson point out that these phrases are not merely metaphorical ways of talking about arguments but rather that the conceptualization and experience of argument itself is based on the idea of war. Their seminal work argues that this process of cognitive mapping across different domains of experience occurs with all different kinds of abstract notions: time as resource ("don't waste my time"), relationship as a journey ("their relationship came at a cross-road"), and theory as a building ("the foundations of your theory are shaky"), among many others.

Over time and with the participation of many scholars, CMT has been expanded to fit alternative variations to the "standard" Lakoff-and-Johnson-style theory. Glucksberg proposes that metaphors act as categorical, class-inclusion assertions - the Categorization View of Metaphor (Kovecses 2013: 12). For example, when someone says "That surgeon is a butcher," what they intend is that that surgeon belongs to the category of 
persons who are butchers in one respect or another (Glucksberg 2008: 68). Conventional metaphors are dependent on preexisting categories (created when the metaphor was first coined) and novel metaphors create new categories where the metaphor vehicle ("butcher") serves as the name of that category (Glucksberg 2008: 69; Glucksberg and Keysar 1998: 422). Another alternative theory is Blending, argued most prominently by Faucconnier. Blending is a conceptual integration theory which argues that beyond the source and target domains of the "standard" theory, metaphors create a new blended domain which exhibits features from both (Faucconnier 2003). In a blended domain, there is a surgeon who acts as a butcher and uses the tools and means of butchery in order to perform surgery on a patient. The main idea behind Blending is that new blended spaces can be used for the generation of new metaphors. Indeed, Faucconnier argues that such integrated conceptual domains can explain most, if not all, of human thought and complexity (Faucconnier and Turner 2003).

It is significant to appreciate these alternative theories because they present a cognitive pattern which differs substantially from the "standard" theory. The arguments of how conceptual metaphor works are arguments of how the mind-and in some research, the brain - works. The "standard" theory posits that cognition works on the basis of projections from one domain to another; the Categorization View holds that conceptual metaphor is a class-creation mechanism; and Blending claims that new mixed or "hybrid" conceptual domains are created through conceptual metaphors.

However, despite the differences between alternative theories, they all espouse the same set of fundamental assumptions that define the "standard" theory. All operate on the premise that there are distinct domains - source and target; concrete and abstract; preexisting and novel - which stand in a particular relationship to each other: movement from the concrete "source" domain to the abstract "target" domain (Steen 2011: 34). They all view metaphor, linguistic or conceptual, as a creative act which combines elements from conceptually — and ontologically — distinct domains. Thus, despite their differences, all of these theories are within the same methodological framework, and can be seen as variations or emendations to the "standard" CMT.

Another key feature in CMT scholarship has been the question of culture. Why do very different linguistic groups have same or similar metaphors? Why do similar linguistic groups have different metaphors? What is the relationship between cognition and culture? These questions have sparked a considerable debate. One key idea that has emerged is embodied realism: fundamental aspects of human cognition are determined by its physicality and environment. There are some conceptual metaphors such as "more is up" and "less is down" which are so basic that, according to Lakoff, they are found in every language and their opposites are found in none (Lakoff 1998: 37). According to this view, CMT has revealed the shared embodied nature of metaphorical cognition, providing a constructive ground for cross-cultural comparative work. On the basis that some metaphors are primary and universal, cultural difference is explained in various ways. One view takes culture as a kind of "sieve" which only allows for some metaphors to pass through. Metaphors which do not cohere with preexisting cultural assumptions do not make it past the "culture sieve" and therefore are not manifested in language (Caballero and Díaz-Vera 2013). Another view grants culture stronger conceptual priority, recognizing that cognition depends on antecedent cultural aspects as much as it does on embodied physicality. A priori views and preferences determined by culture play a constitutive role in the kinds of metaphors generated within a linguistic 
community (Kovecses 2005). The debate has been robust and scholarship on CMT focusing on premodern Chinese texts has had a considerable role in the development of the debate.

\subsection{CMT and Classical Chinese Texts}

To date, the most important work which applies the insights of CMT onto classical Chinese texts is Allan's The Way of Water and Sprouts of Virtue (Allan 1997). Allan's monograph was not only first in analysing Chinese texts on the basis of their shared conceptual metaphors but also the most influential, since it has delineated the contours and modes of scholarship in premodern Chinese literature. However, the most prolific writer in the field has been Slingerland who-with publications spanning nearly two decades - has consistently argued for the application of CMT as an analytical strategy to understand classical Chinese texts (Slingerland 2003, 2004a, 2004b, 2005, 2011, 2017). Combined, their work represents the most authoritative, comprehensive, and original analysis of premodern Chinese texts on the basis of CMT. In both English and Chinese, a growing number of studies reexamining Chinese classical texts under the prism of CMT are being produced (Min 2001; Chen and Holt 2002; He 2003; Reding 2004; Chong 2006; Cline 2008; Giblett 2009; Y. Wu 2011; Lu 2012; Bao 2015; Harrison 2015; Z. Wu 2016). This body of literature touches on a diverse set of texts and explores a wide range of conceptual metaphors.

Methodologically, however, the scholarship shares the same overall approach. The implicit motivation driving the use of CMT in sinology has been an effort to "deexoticize" Chinese classical texts and show that the same conceptual and linguistic mechanisms are in operation in China and the West. For example, reading the argument in the Mengzi 孟子 that human nature resembles water as an example of "corollary thinking" (Jones 2016) stands at odds with a reading that views the argument as a case of conceptual metaphor (Slingerland 2011). While the first reading argues for a uniqueness in the ways reasoning in the text is grounded, the latter construes the argumentative techniques as an example of an ostensibly universal mode of cognition - and in Slingerland's case, Blending as put forth by Faucconnier. Much of the literature has been implicitly driven by an exertion to show that the same conceptual patterns underlie different communities. In her introductory exposition of "root metaphors," Allan draws directly from Lakoff and Johnson to argue for the necessity to study the different metaphorical patterns in different cultures as a means of accessing aspects of fundamental concepts which are "pre-logical" and culturally given (Allan 1997: 10-18). Taking time as an example, she shows how differences in the mythology and general cultural assumptions between the Judeo-Christian and Chinese worlds give rise to very different notions of time. However, Allan takes these very different understandings of time to be metaphorically constructed using the same conceptual mechanism. With CMT in the sinologist's toolkit - the implication is - one can reach a more accurate understanding of the texts at hand, while recognizing shared patterns of thought. Most of the scholarship follows Allan and, no doubt, this body of literature has greatly enhanced the collective understanding of classical Chinese texts and has also done a great deal in "deparochializing" China (Chen and Holt 2002; Giblett 2009; Y. Wu 2011; Bao 2015; Z. Wu 2016). Both of these outcomes are significant and should not be discounted. However, this body of literature has also incorporated the 
assumptions about the validity and effectiveness of using CMT in classical Chinese texts, thus reproducing its inherent cultural embeddedness.

\subsection{Introducing the Problem}

A lot of criticism has been levelled against CMT but none that targets its intrinsic cultural presuppositions. Scholars from various angles have charged CMT on both methodological and theoretical grounds: linguistic evidence based solely on researchers' intuitions, argumentative circularity, faulty taxonomical typologies and many more (Gibbs 2009; de Mendoza Ibáñez and Hernández 2011; Cserep 2014). Targeting the embodied realist position, Rakova reminds her readers that Lakoff and Johnson "forget to show how the visual system can be culturally driven" and therefore "conceptualizing spatial relations ... [comes] prior to the experience of spatial relations" (Rakova 2002: 230-231). Similarly, Link challenges Lakoff and Johnson's claims that certain metaphors are "with a few exceptions" found in all languages by showing how such metaphors do not exist in Chinese, pushing against the implicit norms of what renders a certain metaphor universal (Link 2013: 210).

My critique of CMT, however, is different and in some ways more foundational: the basic problem with CMT is that it has no principled manner to identify "metaphor," be it in language or thought in different cultures. Coming from the Chinese tradition, one wonders whether there is such a thing as "metaphor" in Chinese - a suspicion raised in some classical Chinese literary studies (P. Yu 1987: 10; Yip 1993: 2, 18; Reding 2004: 130). If there is, is it used and understood in the same way as "metaphor" is in modernday English? If there is not, what linguistic tools fulfill similar functions to metaphor in premodern Chinese? In the absence of a clearly identifiable linguistic device analogous to "metaphor," what inferences can be drawn about the "metaphoric" cognitive patterns of the makers of premodern Chinese texts? Answering these questions requires a clear definition of "metaphor" - a point of criticism raised against CMT (Gibbs 2011). However, while this criticism has focused on what constitutes "metaphor" within a linguistic community which already employs the term "metaphor," my critique questions the tacit claim that the very idea of "metaphor" is culturally neutral, namely, that it exists in all cultures and languages. Instead, I want to show that using the term "metaphor" and its derivatives as cross-cultural applications of CMT do is inherently culturally determined and therefore quite likely distortionary.

My critique is about terminology as much as it is about cognition. The point that premodern Chinese does not have a term with a one-to-one correspondence to "metaphor" is not only obviously true but also trivial. The claim is not about the existence of a certain Chinese character but rather about the defining features of intellectual activity in premodern Chinese communities (Hansen 1985: 494). On the one hand, the critique boils down to questioning the assumption of employing the term "metaphor" in premodern Chinese contexts. More culturally neutral terminology is needed: my suggestion is to use the terms "image-based" or "imagistic" language/thought in order to capture all of the linguistic and cognitive phenomena which reveal a tendency to be conceptualized in pictorial ways. On the other hand, challenging the appropriateness of the term "metaphor" can help reveal the ways in which the term from antiquity and up to CMT is informed by particular cultural presuppositions which are not shared with other cultures. "Metaphor" has a particular etymology, history, and theory around it 
which determines the ways in which CMT has been developed. Acknowledging the particularities of "metaphor" allows for a recognition of the cultural embeddedness of CMT and an appreciation of the diversity and plasticity of human cognition. The Chinese etymology, history, and theory around terms placed under the umbrella of "image-based language" point to a different set of cultural assumptions and to a different mode of operation of cognitive patterns. Thus, not only is it necessary to abandon the use of the term "metaphor" but it is also crucial to appreciate its inherent cultural assumptions and the ways in which it has shaped the conclusions of CMT on human cognition.

\section{Drawing Distinctions}

\subsection{Etymologies and Definitions}

"A word never - well, hardly ever - shakes off its etymology and its formation. In spite of all changes and extensions of and additions to its meanings, and indeed rather pervading and governing these, there will still persist the old idea" (Austin 1961: 149). Austin's suspicion of the "trailing clouds of etymology" is captured in the etymology of "metaphor" in the development of CMT. "Metaphor" is derived from the Greek word metaphora, composed by the prefix meta - a prefix with a range of meaning including "together," "with," and "beyond" - and the verb phero, "to carry" or "to bring." In both ancient and modern Greek, the primary and literal meaning of metaphora is "transfer" or "transportation": the metaphora of parcels or means of public metaphora. The very understanding of what it means to express oneself in metaphoric language or to think in metaphorical patterns is itself grounded on the simpler idea of moving one object from one location to another. "Metaphor," be it linguistic or conceptual, is itself metaphorically constructed - a fact seldom recognized in the CMT literature.

The history of "metaphor" starts with Aristotle. "Metaphor," Aristotle tells us, "consists in giving the thing a name that belongs to something else; the transference [metaphora] being either from genus to species, or from species to genus, or from species to species, or on grounds of analogy" (Poetics 1457b; see Aristotle 1932; stress added). Despite the various issues that one can find with Aristotle's definition and set of examples, his understanding of such linguistic tools has exerted tremendous influence over Western thinkers. CMT scholars starting from Lakoff and Johnson are no different, since they tacitly yet fully accept Aristotle's fundamental idea of transferencemappings across different domains. The introduction of a new set of terms should not obfuscate the fact that the same metaphorical construction of "metaphor" based on the idea of movement or transposition determines CMT scholarship and all of its conclusions. CMT literature has of course sought to distance itself from traditional, negative conceptions of metaphor - that it conceals more than it reveals or that it can always be turned into a literal statement. Despite its innovations, CMT has fully remained within the Aristotelian purview since it has not challenged the metaphorical definition of metaphor and instead incorporated the idea of movement as its foundational feature.

The idea of movement, however, is not etymologically present in any of the terms usually rendered as "metaphor" from the classical Chinese locus. If Austin's suspicion 
of the "trailing clouds of etymology" holds true in the Chinese milieu as well, then what does the etymology of the terms which most closely approximate "metaphor" tell us? What are some of the key similarities and differences between the Chinese and Western terminology? What implications do differences in etymology have on the ways in which terms were thought of and used?

In classical Chinese, the words most often associated with (or translated as) "metaphor" - this is conferring the idea of understanding one thing in terms of another not via “simile" or “analogy" — are pi 譬 (comparison) and $y u$ 喻 (explanation). Both of these terms have several meanings. Beyond their function as a kind of literary device, both terms' range of meaning includes actions such as "to explain," "to clarify," "to persuade," "to advise" as well as "to understand" and "to know." In neither of these terms is the idea of transfer or movement from one thing to another present, latently or otherwise. This marks an important difference. It also goes to show that the conceptualization of "metaphor" as a literary device itself metaphorically constructed on the idea of movement is just as - if not more - particular and curious as the conceptualization of "metaphor" qua means of apprehension. Following the intuition of the "trailing clouds of etymology," what the semantic range of the two Chinese terms reveals is that the idea of providing a "metaphor" and the idea of explaining or understanding a certain notion are not seen as two radically different actions. To provide a "metaphor" is to explain and to explain is to provide a "metaphor."

It should be rather apparent then that if one were to develop a theory of cognition based on the etymological background of the classical Chinese linguistic locus, the idea of movement and transposition would be rather odd and hard to articulate. Rather, it seems to me that such a theory would more likely be based on the conceptualization of image-based thinking as a more or less direct and undeviating mode of understanding. If CMT were to inductively reach its conclusions from the "bottom up" - that is, taking primary evidence and inspiration from as many cultures as possible to formulate its theoretical building blocks - then it would have to seriously reflect on the universality of the idea that cognition works as a series of conceptual transpositions across domains. The absence of the idea of movement from classical Chinese suggests that this understanding of image-based language is particular to one culture and constitutes an intellectual imposition when applied to other cultural systems.

\subsection{What Metaphor and "Metaphor" Are Not}

The ways in which the idea of metaphor or $p i$ and $y u$ have been constructed in either tradition do not solely depend on how the terms have been defined but also on how they have been understood to be different from other terms. What these words mean not only rests on what they are but also on what they are not - and such distinctions have had a tremendous impact on the development of these terms in both traditions and on the formation of CMT itself.

In the Western discourse, metaphor has been understood as qualitatively distinct from simile and analogy. Simply put, a simile has a construction which exhibits the fact that it connecting two different objects. Because of this difference in its morphology, even though the propositional content between a metaphor and a simile may be the same, the two have been understood to be qualitatively distinct. Similarly, analogy is the link between two objects based on similarity or proximity (Bybee 2010: 57). In either case, 
metaphor has been privileged as unique either in its ability to conceal or reveal (Kao 2000: 4; Kallay 2014: 342). Historically, the dominant stance toward metaphor - at least in philosophical discourse - has been negative since it has been seen as a rhetoric method which obfuscates meaning. ${ }^{2}$ CMT has argued against such view and instead has maintained that metaphor at the conceptual level has been the driving mechanism behind all kinds of cognition. For better or worse, metaphor has been seen as a special device which operates in quite distinct ways from either simile or analogy.

The uniqueness conferred to metaphor has influenced CMT in two ways. First, CMT has fully accepted that metaphor stands in a privileged position over other linguistic tropes since its method of constructing similitude between two objects intimately reveals the cognitive patterns that underpin all metaphoric language constructions. The fact that metaphors do not present themselves like similes or analogies, is, for CMT, a reflection of the fact that most of our abstract thinking depends on implicit projections from one domain space to another. The reason people are generally unaware of the prevalence of metaphors in language and their lives is because metaphors are by their very nature hidden since, unlike similes, they do not employ special linguistic markers. The element of hiddenness partly explains why mappings between domains (i.e., conceptual metaphor) has not been noted in the past. Second, CMT has been influenced by the historical debates on the value of metaphor, since it quite actively takes a position within the debate. CMT has argued for a new understanding of metaphor which renders it ubiquitous and necessary, and by extension positive since it is due to conceptual metaphor that art, scientific discoveries, and all kinds of abstractions are based. Thus, even though CMT often presents itself as radically different from past conceptualizations of metaphor, it carries much of the theoretical baggage attached to metaphor historically.

In classical Chinese there are distinctions between different literary devices but they are of a fundamentally different nature. The first distinctions in literary devices can be found in the "Great Preface (Daxu 大序)" of the Classic of Poetry (Shijing 詩經; both texts canonized in their received forms around the 1st c. BCE), the earliest and most influential collection of poems from antiquity. Both the "Great Preface" and the Classic of Poetry have unclear origins and their received versions are the accretion of various scholars' writing over time. Both texts were considered invaluably significant - as the appellation "classic" shows - and were part and parcel of scholarly education. As such both exerted a tremendous influence over Chinese poets, literary critics, and literati at large, delineating the contours of scholarship much in the same way as Aristotle's definition and elaboration of "metaphor" has done in the Western tradition. The "Great Preface" identifies three literary devices as the main mechanisms through which poems express their meaning: $f u$ 賦, $b i$ 比, and xing 興. The first term denotes “exposition” and can be considered as a standard form of narration - in the eyes of traditional Chinese commentators, this term appeared to be the most straightforward and least problematic. It was the disambiguation between $b i$ and xing, and the appraisal of the two, which stirred most literary debates ( $\mathrm{Gu}$ 1997). Xing is most often rendered as "evocation" or

\footnotetext{
${ }^{2}$ Of course, attitudes toward linguistic metaphor have differed greatly over time and over different fields of learning. By no means is the short presentation of the historically perceived function and merits of metaphor supposed to be complete or exhaustive. I merely follow CMT literature in its presentation of the history of metaphor simply to remain within the same discursive contours.
} 
"affective image" and its goal is to create a mental image of a certain scene without connecting it to a particular object and to produce a particular emotive reaction to the reader. On the other hand, $b i$ - which also means "closeness" or "proximity" - comes close to the idea of "comparison" where one object is compared to another. Depending on the context, bi can be translated as either "metaphor," "simile," or "analogy" (Owen 1992: 46). Coupling bi with $y u$ gives the modern Chinese term for "metaphor."3

The ways in which $b i$ has been conceptualized has heavily depended on the ways in which it has been perceived as different from xing. The Literary Mind and the Carving of Dragons (Wenxin Diaolong 文心雕龍; composed in the 5th c. CE) is the first and among the most influential works of literary criticism in the Chinese tradition. It was written in an explicit conversation to the "Great Preface" and one of its chapters explains in great detail the difference between bi and xing. The text identifies a distinction between the two in form and function with clear ramifications on the value between the two. According to The Literary Mind, bi is direct and explicit while xing is oblique and latent. It is quite easy to understand the connection made between two parts of $b i$, but it is harder to pinpoint what affective reaction xing is intended to create. For this reason, the text argues that xing is superior to $b i$, since it strongly outweighs $b i$ in subtlety and evocative power (S. Zhang 1993: 206-207).

As a standardized literary tool, xing does not have an analog in Western literature since there is no trope which aims solely to evoke a particular image or stir a certain emotion without drawing a metaphoric link to a particular object. The uniqueness of xing as a form of expression and thought shows that the conceptual environment in which $b i$ was developed is significantly distinct from the one from which metaphor grew. Even if $b i$ and metaphor do have some overlapping semantic ground, they both retain essential differences, as revealed by the fact that these terms were understood in opposition to substantially distinct objects (xing v. simile or analogy). Therefore, translating $b i$ as "metaphor" runs the double risk of concealing important nuances and of imposing an alien conceptualization.

Again, these points of difference seem to indicate a different route for a theory of cognition. CMT has continued the Aristotelian discourse which privileges metaphor among literary devices. However, a theory of cognition derived from classical Chinese texts would most likely pay more attention to xing as the most meaningful manifestation of image-based language and xing does not purport to link one object with another. In such a theory of cognition, "metaphor" would not even have the most prominent role, since it pales in comparison to xing according to traditional Chinese scholarship. Last, even if "metaphor" occupied a dominant role in a Chinese-derived theory of cognition, the theoretical mechanisms providing the basis on which a comparison is drawn are different between $b i$ and metaphor.

\footnotetext{
${ }^{3}$ In modern Chinese, biyu 比喻 is the standard translation for metaphor: biyu exists in classical Chinese but fixing its meaning to fit "metaphor" has been a linguistic innovation undertaken during China's early stage of modernization in the beginning of the 20th century. This point is illustrated by the fact that literary scholars and dictionaries at the time provided different combinations of Chinese characters in order to render the term "metaphor" (Cai 1993: 233). The fact that there is one definition of "metaphor" in modern Chinese is not because such a term naturally emerged from the tradition but rather because a concerted and systematic effort was made to create a new modern language which would be able to express Western ideas. This process of term creation, of course, was not limited to literary devices but instead permeated all fields of knowledge (Makeham 2012).
} 


\subsection{Theoretical Investigations}

Unlike metaphor, $b i$ is not understood as establishing a new link between ontologically distinct objects or domains. $B i$ is merely a form of comparison between two objects premised on the familiarity of a language community or group with these two objects. In the "Appropriate Sayings (Shanshuo 善說)" chapter of The Garden of Sayings (Shuoyuan 說苑; compiled in the 1st c. BCE), we find a crucial idea: the use of "metaphoric" language depends on the ways in which a community relates to its objects of discussion. The passage is an exchange between a king and his retainer. The king asks for "direct language" without the use of "analogies" or "metaphors" in order to explain things. The retainer replies that without recourse to such tools, explaining what a tan 彈 (crossbow) is (i.e., a new and unknown object) would confine one to saying that "a tan is like a tan." With nondirect language, however, one can say that a tan is like "a bow but with a bamboo string" and with such a description, the courtier argues, one can understand what a tan is. The king agrees and the courtier concludes that one "always uses what it known to explain what is not known" (Ekstrom 2014: 53). ${ }^{4}$ The interesting point in this exchange is that the premise of "metaphoric" language does not rest on some assumed preexisting similitude between the two objects but rather on the relationship that the community or group of language users has to the objects in question. Of course some kind of similarity exists between a tan and a bow for the "metaphoric" explanation to be meaningful. However, the salient point here is that the courtier's argumentation is not premised on the idea of similitude between the two objects but rather on the group's relationship to them. This conceptualization of "nondirect language" places very little emphasis on the "actual" degree of similitude between two objects, locating instead the establishment of "metaphoric" language on the degree of familiarity with an object. It does not matter how objects are - because, in the traditional Chinese worldview, they are always in a state of changebut rather how objects are perceived. In the Western context, however, metaphor rests on different theoretical assumptions.

Metaphor depends on a metaphysical outlook which assumes that certain words "properly" apply to certain objects (i.e., the "literal" level of signification). Metaphor is the transposition of such "literal" words from one object to another. Aristotle's theory of metaphor could not be produced had he not inherited a robust linguistic realism that certain words "belong" to certain objects. In metaphor the act of transplacing one word from one object to another exists because of an antecedent belief in the link between word and object. The possibility of metaphor depends on the similarity that can be found between two objects as it exists independently of the language community.

Again it is easy to believe that CMT has distanced itself from the Aristotelian conception of metaphor since almost all CMT scholars do not hold that conceptual metaphors reveal an antecedent metaphysical structure about the world but are rather specific sociocultural creations of language communities and individual language users. Nonetheless, CMT has a robust and nontrivial set of metaphysical assumptions. The division of the world - physical or conceptual - in domains such as "concrete" and "abstract," "simple" and "complex," "physical" and "human"- to which "target" and "source" come to be appended—requires a set of

\footnotetext{
${ }^{4}$ The text uses the term $p i$ 譬 to denote the idea of "analogy" and in this context it is very similar to $b i$. The terminological fluidity of classical Chinese is another feature which would significantly influence the ways in which a theory of cognition derived from premodern Chinese texts would look.
} 
substantial assumptions. The first assumption is that the world is indeed divisible. Then it is assumed that the way in which it is divisible coheres with a theory of essential properties: objects are placed together in the same domain because they share a property that distinguishes them from all other kinds of objects. These domains or categories are definable, enclosed, and delineable. Third, CMT assumes that these domains can interact (at the very least in our minds); and four, that this interaction happens only in one direction from the "concrete" and "simple" "source" domain to the "abstract" and "complex" "target" domain.

Even if CMT scholars argue that their division of the world does not have any metaphysical aspirations, it is still reflective of a particular way of thinking paradigmatic in the West. The fact that the division of the world in domains such as "concrete" and "abstract" or "physical" and "human" appears so intuitively true to a modern Western reader does not show that the statement is accurate but rather reveals the extent to which such a way of thinking about the world is culturally engrained. The division of the world is not an independent, neutral fact but is rather a specific cultural construction that determines the ways in which the world is understood and experienced. The division is not universal but rather particular and CMT cannot be established without this antecedent worldview - be it metaphysical or not. Finally, it is not evident that the argumentative strategy of avoiding metaphysics is open to CMT, since it cannot coherently maintain its position of conceptual metaphor as a movement from one domain to another without any metaphysical claim whatsoever. Heidegger's statement that "the metaphorical obtains only with metaphysics" goes to show the inherent link between the notion of metaphor-linguistic or conceptual - and a specific metaphysical worldview (Heidegger 1957: 89). It is just not possible to have an idea of metaphor, and language by extension, without a particular metaphysical outlook. Thus even though CMT scholars do not want to engage with metaphysics they have not found a way to extirpate the metaphysical baggage attached to metaphor.

In the classical Chinese setting, a discourse on "metaphor" based on the idea of transposition of terms which "properly" apply to a particular object or domain did not - and could not - take place. Objects in the Chinese understanding are not discrete entities with stable identities bur rather open-ended processes undergoing nonending change (Ekstrom 2014: 50). Since there are no fixed identities, words cannot "belong" to certain objects and metaphor cannot be conceptually established since the idea of "borrowing" presupposes an idea of stable "ownership" in the first place. For example, a "metaphor" between water and sagacity in classical Chinese is not understood as a mapping from one "concrete" source domain (water) to one "abstract" target domain (sagacity). Instead, water and sagacity are conceptually perceived as being part of the same cluster of things (lei 類) determined by a similar pattern. These clusters, however, are not mutually exclusive and are defined by dynamic interactions between and across their constitutive members (P. Yu 1987: 41-43). Such fluidity in the way in which the world was conceptualized helps one understand a key aspect of Chinese critical terms - that they do not correspond to a single and clearly definable concept (Liu 1975: 13). ${ }^{5}$ There are no distinct domains, there is no fixed demarcation between "concreteness" and "abstractness." Water and sagacity are part of the same grouping

\footnotetext{
5 This is the reason I have chosen to translate lei not as "category/categorize" as is commonly done but rather as "cluster." The problem with the term "category" is that it implies a definitional and fixed structure pinned on the idea of mutual exclusivity that is not present in the use and understanding of the term lei.
} 
and the conceptualization of either one informs the other: water structures the idea of sagacity but sagacity also structures the ways in which water is conceptualized. It is for this reason that "naturalistic" traits are ascribed to human features and vice versa that "anthropomorphic" descriptions are given to natural elements.

A fundamental aspect of the Chinese worldview - permeating all schools of thought and texts - is the idea of unity between the human and natural worlds (tianren heyi 天人 合一). This theoretical outlook of the world is significantly different from the metaphysical presuppositions that underpin most of Western philosophy, theories of metaphor, and CMT itself. In this view, the world is not divisible in discrete categories. Objects within the world are located in the same "existential plane" without sharp ontological divisions between the "human" and the "natural." With such an outlook, it is almost impossible to develop a conceptual structure like metaphor which presupposes clear ontological divisions. Furthermore, tianren heyi has important implications about the nature of the relationship between world and language.

The structure of one of the most influential texts from Chinese antiquity, the Classic of Changes (Yijing 易經; compiled around the 1st c. BCE), reflects - and constructsthe foundations of premodern Chinese beliefs toward world, image, and language. The mythological origins of the text are combined with a traditional narrative about its development which illustrates dominant conceptions about the relationship between world and language. The Classic of Changes, tradition tells us, started with pure observation of the world and its patterns of change. From those observations, the mythological sages of antiquity derived images - sets of broken and unbroken lines representing the two main forces of change in the world, the yin 陰 and the yang 陽一 which describe the fundamental patterns of change. However, in light of the need to explain the images, textual commentaries were appended elucidating the meaning of each image. Regardless of its historical accuracy, the interesting point about the traditional narrative of the formation of the Classic is that it sees a natural evolution from world to language, mediated by images. Language is held to be derived from these graphic representations of the patterns of change, which are themselves derived from the patterns of change of the world itself: there is no sharp division between the "physical" and "human" realms. The conceptual link between world, image, and language can further be seen in the etymology of the term wen 文 which means both "pattern" and "language"/“literature" (Liu 1975: 7; P. Yu and Huters 1994: 21; L. Zhang 1996: 21; Hurst 2000: 11). ${ }^{6}$ What does all this mean for 'metaphoric' language?

The Principles of Literature (Wenze 文則; composed in the 12th c. CE) is the first systematic study of rhetorical tools in the long history of literary commentary in China and among its most influential. The text acknowledges the inherent link between world, image, and language and argues for the necessity of "metaphoric" expressions: "The Classic of

\footnotetext{
${ }^{6}$ Notice that if coming from a Western point of view it is easy to mislabel the coextension of the linguistic world with the natural world as an example of linguistic realism. This is not the case because linguistic realism (under one dominant understanding at least) presupposes and indeed is the fixity of names to specific ontological essences. The idea in the Chinese setting of coextension of language and world rather rests on the recognition that change is the modus operandi of both the physical and human realms. Furthermore, as already shown at the beginning of this subsection, discussions about the appropriateness of language in Chinese classical texts evince little, if any, concern for their relation to the natural world. Instead they almost always focus on the social dimensions and implications of the use of "proper language," as can be seen in the discussion on the "Shanshuo" above or in Confucius' much quoted passage on the rectification of names (zheng ming 正名).
} 
Changes uses images (xiang 象) to express its meaning; the Classic of Poetry uses 'analogies' ( $b i$ 比) to express its sentiment. How can works of writing not have 'metaphor' ( $y u$ 喻)?" (Cai 1993: 67). Yu - that is, image-based language - is necessary in expressing oneself because of its inherent link to the world. Because $y u$ is based on an image-based understanding of the world, it comes closer to describing it. Not only is $y u$ not seen as a problematic or concealing tool but it is privileged in its access to reveal facts about the world exactly because it is pictorial in nature. With this conception in mind, the classical Chinese tradition did not perceive image-based language as an act of creation on the part of the poet or thinker but rather as an act of revealing a preexisting link between different objects. "Metaphors" are not the products of a creative process but rather discoveries about fundamental aspects of the world (P. Yu 1987: 65). Appreciating this point helps one understand why classical Chinese texts brought together issues of cosmology, governance, ethics, and aesthetics, for they were seen to exist in the same plane. The dominant view in Western thought has been quite different.

Metaphor has been conceived as a form of artistic creation following the pattern of Platonic mimesis (imitation). An artistic representation of an object, linguistically or graphically, only partially and imperfectly represents an object. Moreover, metaphor as an example of linguistic art has been conceived as the product of a creative process. Uttering a metaphor is not the result of a process of discovery of preexisting patterns but rather the product of a creative process. Thus, even though objects are seen as having an "actual" affinity between them, metaphor is conceived as the end-product of a creative mind.

CMT has incorporated this aspect of creativity in the formation of both the "standard" and alternative theories. CMT scholars are overwhelmingly concerned with how conceptual metaphors come to be as objects of creation by individuals or communities. Lakoff and Johnson, and the literature that follows their line of reasoning, have aimed to explain how "similarities are created," while both the Category Construction and Blending views assume a high degree of creative engagement in the utterance of metaphor. A theory of cognition derived from premodern Chinese use and reflection on image-based language, however, would most likely not focus on the point of creativity at all. Instead, it would more plausibly aim to show how cognitive patterns resonate with patterns of change in the world, in turn seen as further evidence of the coherence of the unity of the "human" and the "natural."

\section{Further Clarifications}

\subsection{One Size Does Not Fit All}

Defenders of CMT often point out that its critics fail to recognize how the theory has developed and matured over the past three decades or what alternative to the "standard" theory have been proposed. Most critics begin and end their engagement with CMT with Lakoff and Johnson's seminal work (Gibbs 2009: 17). I hope it has become apparent from the previous sections that this essay has engaged with far more material from the CMT literature. Nonetheless, in this section I want to explore the fundamental issues with using CMT in the premodern Chinese context by closely examining a set of claims about the cognitive patterns of the makers of 
classical Chinese texts as presented by Slingerland, the most prolific scholar in the field. His specific position does not represent every scholar in the literature. However, his attitude of using in toto and without modification the conclusions of CMT, or one of its variants, is representative of the prevailing way of thinking in the field: even if different scholars subscribe to different theories of conceptual metaphor, they all apply them onto the makers of classical Chinese texts without qualification. Slingerland's work is wide-ranging, raising interesting - if sometimes debatable - points on a number of issues. For reasons of clarity and brevity, I will only focus on five key points taken from a set of his most representative work, and I will (preemptively) respond to three objections that can be detected in his work and the CMT literature at large. The critique toward Slingerland's work can be extended toward CMT in general.

\section{Point \#1: Broad Definition of Metaphor}

Below I will argue for a fairly broad conception of metaphor as the use of one, usually concrete, domain to structure our understanding of another, usually more abstract, domain. "Metaphor" understood in this sense includes what we might otherwise label as analogy or allegory. This is roughly the understanding of metaphor that informs most of the work on metaphor in early China. (Slingerland 2011: 2)

It is evident that Slingerland is concerned exactly with the question that motivates this essay: does "metaphor" exist in classical China? Slingerland provides this definition of "metaphor" which he qualifies as being "fairly broad," which indeed it is, if judging from a Western literary standpoint. However, no matter how thinned down the conception of metaphor may be, it still retains fundamental aspects of its etymological and metaphysical construction which do not render it a culturally neutral tool of analysis. The foundational idea of transposition along a robust set of metaphysical commitments to the divisibility of the world in delineable domains is the theoretical prerequisite for any theory of conceptual metaphor. Stretched, cut, or flattened out, metaphor retains its theoretical baggage, determined by a specific cultural experience-metaphor is not as innocent of an idea or methodological tool as Slingerland would suggest. Image-based language as captured by the terms $y u$ and $b i$ among others has a different etymological and theoretical background not coherent with the idea of transposition and of delineable domains.

\section{Point \#2: Embodied Realism}

Because human bodies are quite similar the world over, and the types of environments human beings face are also shared in most important respects, one would expect to find a high degree of similarity with regard to conceptual metaphors across human cultures and languages, especially with regard to primary metaphor. (Slingerland 2004a: 327)

Metaphor, then, can serve as a linguistic "sign" of otherwise inaccessible, shared, deep conceptual structure. (Slingerland 2004a: 336) 
Slingerland is concerned with showing that the same embodied reality of human beings determines the most fundamental aspects of human cognition. In principle, I do not disagree with this claim and I believe that indeed a shared level of cognitive mechanisms can be found between premodern Chinese communities and modern-day users of English. My claim rather is that current theories of conceptual metaphor do not fulfill this function because such theories have not been formed on the basis of empirical, linguistic, and theoretical evidence from as many cultures as possible. Instead, CMT and its variations have been premised exclusively on the Western experience of and reflection on metaphor, a culturally loaded term to begin with. Instead of imposing concepts and schemata which have been derived only from a certain cultural background in order to describe that background, we have to accept - indeed invite - a radical questioning of key concepts and schemata as informed by the experience of crosscultural contact. An inductive construction of new theories working from the "ground up" is needed. So long as such kind of methodological ethos does not take hold, academic research will be stuck in reproducing the fallacies of a Western universalistic discourse as it has in the past, be it in the form of Jesuits' reading Christianity in the Chinese classics, Marxist periodization of Chinese history, or the use of CMT to describe the cognitive patterns of premodern Chinese thinkers. There is a shared cognitive foundation throughout humanity, but conceptual metaphor is not it.

\section{Point \#3: Universal Metaphors}

As we shall see as we begin to apply the methods of cognitive linguistics to classical Chinese texts, the degree of similarity we will find between modern English and ancient Chinese conceptual metaphors is quite striking, even when it comes to quite abstract and presumably culturally contingent domains. (Slingerland 2003: 26)

A direct corollary of embodied realism is that some metaphorical structures of cognition are found virtually in all cultural and linguistic communities because they stem from some very fundamental and basic facts of human bodies and their interaction with the physical environment. This statement is problematic even if the problems with identifying conceptual metaphor as the shared foundational cognitive process are ignored. Examples of primary metaphors are "more is up" and "less is down," and "accompaniment as instrumentality" (for example, "cut with a knife") which according to Lakoff are found in all languages save a few exceptions (Lakoff 1998: 37). First, it is obviously not the case that all cultures share these primary metaphors since there are many counter-examples in the literature. For classical Chinese in particular, the conceptualization of Dao 道—also thought of as the highest good — as a low-lying body of water challenges the claim of universality of the "more is up" and "less is down" structure (Chen and Holt 2002; Giblett 2009; Lu 2012). Furthermore, Link has also shown that Chinese (both premodern and modern) is one of these "exceptions" when it comes to the "accompaniment as instrumentality" metaphor (Link 2013: 210). Second and more importantly, one cannot but wonder how CMT scholars have established their norms for universality and how they have been testing for them. If, for example, hundreds of languages shared a metaphor but Spanish, Arabic, and Chinese did not (representing almost half of the world population) then would such a metaphor be 
deemed universal? The suspicion is that what are seen as universal primary metaphors simply are the metaphors found in English which are then plausibly detectable in a handful of other languages.

\section{Point \#4: Metaphysical and Cognitive Convergence}

The metaphysics built into the Zhuangzi’s [莊子] classical Chinese metaphors resonates strongly with the (mostly unconscious) metaphysical assumptions built into the metaphors of modern American English. (Slingerland 2004a: 322)

The blends constructed by the author of the Mencius are re-created by our own brains as we read the translated text. (Slingerland 2005: 581)

However, the argument does not follow for several reasons. First, one would have to accept that Blending (or any other variant of CMT) is indeed the mechanism by which human beings, at the very least today, think. This is by no means an established fact. Second, even accepting that living people today have a cognitive mechanism based on Blending, it would take a significant conceptual leap to show that a community which understood language and "metaphor" and its uses in radically different ways had exactly the same pattern of cognition. Slingerland mistakenly assumes that the fact that Chinese classical texts can be analyzed in a way which more or less appears to cohere with Blending is a sign of a shared pattern of cognition between the makers of the text and its modern readers. Just because a certain object or phenomenon can be explained in a certain way does not mean that such description is accurate. For example, the geocentric view of the universe was firmly believed not only because of the strong impression of planetary rotation around the earth but also because it worked as a system on which fairly accurate predictions about the motions of heavenly bodies could be made. Just because a certain theory can explain an object or even make predictions for it does not mean it is an accurate description of that object. Slingerland takes Blending to be a fitting description of the texts and the mental processes behind their formation, failing to recognize that it is an interpretation.

\section{Point \#5: Methodological Benefits}

The cognitive-linguistics approach thus not only represents a powerful and concrete new methodology for cross-cultural comparative work but also provides us with a convincing and coherent theoretical grounding for the comparativist project itself. (Slingerland 2004a: 336)

The cognitive linguistics approach has indeed liberated the crosscultural study of ideas and social phenomena from the clutches of an overly modernist, "West-is-best" discourse. It has breathed fresh air to the decolonization project, helping to understand and appreciate thought from communities around the world. Nonetheless, cognitive-linguistics - in its manifestation in CMT at least - should not be seen as a completely bias-free enterprise. The notion of conceptual metaphor is informed and determined by a very specific set of sociocultural and linguistic assumptions which are found only in one culture. Using conceptual metaphor as the foundation for comparativist projects is neither convincing nor coherent, for CMT literature has neither shown that conceptual 
metaphor is a culture-neutral analytical tool nor that it is aware of the inherent cultural assumptions underpinning conceptual metaphor. Of course, Slingerland is not alone in this line of reasoning. Indeed, almost all of the literature which applies the conclusions of CMT on premodern Chinese texts incorporates the same assumptions. The only difference between Slingerland and other authors is that he is more explicit in his conclusion that conceptual metaphor was indeed the cognitive mechanism by which premodern Chinese operated. Other scholars, however, are no less committed to the idea that the cognitive mechanisms described by CMT apply to the makers of ancient Chinese texts. Beyond the literature on classical Chinese, the vast majority of crosscultural CMT research has assumed that conceptual metaphor can be detected in all languages and attributed to all communities. As such, I would like to respond to three points of critique that can be levelled against this essay.

\section{Objection \#1: Focus on Image-based Language}

A first objection against this essay could be that my focus and understanding of metaphor has been too narrow. Such an understanding of metaphor, obviously, does not exist in the premodern Chinese setting. That is the reason why a lot of the literature uses the terms "imagistic" or "image-based" language to ascertain the cognitive phenomena underpinning these expressions. It is true that a lot of scholarship prefers the use of these terms. Nonetheless, it is unclear what this shift in terminology amounts to methodologically. Since research on conceptual metaphor in the classical Chinese setting has not questioned the very existence of metaphorical thinking, it is unclear how the conclusions of CMT are not applied without qualification to the makers of ancient Chinese texts. Use of new terminology would be meaningful if accompanied by a reflection on the use of the term "metaphor" just like literary scholars of classical Chinese literature have done (P. Yu 1987; Reding 2004). Conceptual metaphor research may be using more culturally neutral terms but the theoretical content behind these terms is no different from scholarship, like Slingerland's, which uses the terminology and theoretical framework of CMT without qualification. The use of new terminology has to be combined with a stronger element of self-reflexivity and with a desire to develop a culturally broader theoretical framework which takes its evidence from as many cultural backgrounds as possible. Using new terminology without an accompanying change in methodology and theory is not meaningful change.

\section{Objection \#2: No Word $=$ No Concept}

This objection can be understood as a challenge to the so-called "Whorf-Sapir" hypothesis that if a language does not have a word for a concept then it lacks that concept altogether. This is a broad and ongoing debate with evidence growing for both sides of the debate from various fields coupled with new theoretical analyses. I hope that this essay has sufficiently shown that both metaphor and $y u, b i$, and other Chinese terms do not exist in a linguistic and theoretical vacuum but rather in a constellation of terms steeped in specific assumptions about the world, language, and their relationship. I hope it has also been made clear that even the broadest understanding of metaphor does not cohere with the evidence of the use and reflection upon image-based language in the Chinese setting. Furthermore, to say that the premodern Chinese did not have a concept of metaphor opens up the space 
to ask what ideas they did have. From that foundation, theories on cognition can be derived from classical Chinese texts and help broaden the collective understanding of human thought. To say that classical Chinese does not have a word for metaphor is not a claim only about a rather obvious linguistic fact but rather a call to try and understand premodern Chinese texts "on their own terms" (Hansen 1985: 494; Kao 2000: 7). Premodern Chinese does not share even the most expansive understanding of metaphor. Instead, it has its own rich and diverse theoretical environment in which different terms denoting different ideas existed and developed.

\section{Objection \#3: Chinese Exceptionalism}

Many scholars within sinology take this claim with a modicum of offense, since Chinese uniqueness is often equated with an exoticizing discourse of inferiority. To a large extent, the early stages of modern and contemporary sinological research have been marked by an exceptionally negative attitude toward Chinese thought, consistently presenting it as weak, mangled, and sophomoric compared to an idealized and ultrarationalist ancient Greek discourse. I understand where scholars committed to showing convergence between China and the West are coming from and I recognize and value their intentions. However, a quest for commensurability cannot be an end by itself, lest commitment to accuracy be sacrificed. More important than showing similarity between the two traditions is fully liberating scholarship — not just within sinology — of the strong negative biases against Chinese thought. There are many possible routes to achieve this, but resorting to forced commensurability is not only inadvertently misleading but also plausibly detrimental to the goal of producing a globally inclusive dialogue. If every community is reduced to being perceived as having the same linguistic and cognitive patterns as the West, it then becomes unclear how different experiences can work as an object of reflection and enrichment for all. Diversity — as the cliché goes — should be embraced, not feared.

\subsection{Cross-Roads and New Paths}

Should CMT then be discarded for studying premodern Chinese texts, and by extension texts from other cultural traditions? Are the conclusions of CMT invalid in non-Western cultural contexts? What is the role of conceptual metaphor in crosscultural research? Despite the strong critique developed in this essay, I want to provide a brief account as to why CMT should not be abandoned but rather reconceptualized-for I believe that, if properly modified or qualified, CMT can be useful in analyzing premodern Chinese texts. There are two different approaches at hand. The first one calls for a reevaluation of conceptual metaphor on the basis of new evidence as put forth by the premodern Chinese locus. The second calls for a reexamination of the function of CMT, moving it from the grounds of description to the field of interpretation.

The first approach requires systematic and sustained effort from scholars from various disciplines (and cultures) aimed at broadening the experiential and theoretical basis on which CMT is premised. How does the use of image-based language in different cultural groups, and their reflections on such use, come to affect the ways in which CMT is developed? How are the ideas of "metaphor," "domain," "mapping," "blending," and so forth changed when examined from different cultural spheres? Can 
these terms survive albeit requalified? Or do they need to be replaced by new and broader terminology? It is not possible within the scope of this essay to show what such a revision would look like. Rather, I hope that by raising these questions other scholars will start questioning the conceptual validity of extrapolating on the cognitive processes of diverse cultural communities on the basis of the assumptions of CMT.

The reason CMT can still be helpful in understanding classical Chinese texts is that it is an improvement on existing methodological tools. Save but few basic metaphors, CMT does not purport that the same conceptual metaphors apply in all cultures, but rather that each culture thinks "metaphorically," that is, in terms of imagery. Imagebased language is indeed found in premodern Chinese texts and arguably all languages of the world reflect on some level a shared pattern of expression and cognition. What I am skeptical of, is that such universal pattern is conceptual metaphor, since this would require a broad, deep, and very specific set of assumptions. Nonetheless, some kind of image-based thinking following a presently unclear mechanism does seem to exist. The benefit of using CMT then lies on the level of linguistic signification, since using the methods of CMT to understand the imagistic construction of meaning in the texts is indeed beneficial, and no doubt an improvement compared to past practices imposing alien image structures or concepts. Revealing and appreciating the imagistic entailments in meaning helps come closer to classical Chinese texts. Through this method, a fairer reading is given to texts, something usually not afforded by methods derived from, for example, analytic philosophy. However, the functionality of CMT on the level of meaning construction should not be extended to speculations on underlying patterns of cognition, no matter how robust the understanding of conceptual metaphor is. Hopefully, it has become apparent that there is sufficient evidence to ground a healthy skepticism toward such speculations and to explore the possibility of unearthing more fundamental patterns of image-based cognition.

In tandem with the first approach, the second line of reasoning calls for CMT to take a more nuanced position toward its objects of explanation, at the very least for communities for which it cannot falsify its claim with experimental evidence from the fields of psychology and neuroscience (i.e., nonliving communities). Claiming that conceptual metaphor underpins all methods of cognition across time and space is a universalistic discourse bent on showing - or rather creating - a world patterned on the experiences and reflections of only one particular group. As long as the primary constitutive elements of CMT are not actively derived from as many linguistic communities as possible, the specific conclusions of CMT remain bounded by cultural specificity. As such, CMT can be viewed as a helpful interpretative tool for texts from antiquity, placed in between the object of study and the community conducting the study. CMT is not an accurate description of the cognitive mechanisms of the premodern Chinese but rather a tool applied to the text in order to make it understandable to the community of researchers in question. In this sense, CMT has to be seen as a bridge between the object and subject of study and not as a faithful reconstruction of cognitive patterns allegedly at work in the minds of the makers of these texts. While recognizing the interpretative possibilities of CMT, it is essential to steer clear of a hegemonic discourse which indiscriminately applies theoretical categories and value judgements. It is even more crucial to acknowledge this in light of the fact that CMT quite often presents itself as culturally neutral. 
Moving from a discourse based on metaphor to one based on image will require a broadening of the evidential pool, along with a thorough reconsideration of the most important theoretical conclusions of CMT. I am hopeful that such a move canactually, will - happen, as more scholarship becomes dedicated to show the cultural embeddedness of conceptual metaphor. CMT has meaningfully contributed to the understanding of meaning creation in ancient and culturally distinct texts. It can now meaningfully contribute to the understanding of cognition if it questions its fundamental assumptions.

Open Access This article is distributed under the terms of the Creative Commons Attribution 4.0 International License (http://creativecommons.org/licenses/by/4.0/), which permits unrestricted use, distribution, and reproduction in any medium, provided you give appropriate credit to the original author(s) and the source, provide a link to the Creative Commons license, and indicate if changes were made.

\section{References}

Allan, Sarah. 1997. The Way of Water and Sprouts of Virtue. Albany: State University of New York Press.

Aristotle. 1932. Aristotle in 23 Volumes. Trans. by W. H. Fyfe. Cambridge, MA: Harvard University Press. Perseus Digital Library. http://www.perseus.tufts.edu/hopper/text?doc=Perseus\%3atext\%3a1999.01.0056 (last accessed on May 14, 2019).

Austin, John L. 1961. Philosophical Papers. Oxford: Clarendon Press.

Bao, Yongling. 2015. "Water, Plant, Light, and Mirror: On the Root Metaphors of the Heart-Mind in WANG Yangming's Thought." Frontiers of Philosophy in China 10.1: 95-112.

Bybee, Joan. 2010. Language, Usage and Cognition. Cambridge: Cambridge University Press.

Caballero, R., and J. Díaz-Vera. 2013. "Metaphor and Culture: A Relationship at a Crossroads?” Intercultural Pragmatics 10.2: 205-207.

Cai, Zongyang 蔡宗陽. 1993. A New Study on CHEN Kui”s “Principles of Literature” 陳騤《文則》新論. Taipei 台北: Wenshizhe chubanshe 文史哲出版社.

Chang, Miao-Hsia. 2015. “Two Counter-expectation Markers in Chinese.” In Metaphor and Metonymy Across Time and Cultures: Perspectives on the Sociohistorical Linguistics of Figurative Language, edited by Javier Diaz-Vera. Berlin: De Gruyter.

Chen, Guo-Ming, and Richard Holt. 2002. "Persuasion Through the Water Metaphor in Dao De Jing." Intercultural Communication Studies 11.1: 153-171.

Chong, Kim-Chong. 2006. "Zhuangzi and the Nature of Metaphor." Philosophy East and West 56.3: 370-391.

Cline, Erin M. 2008. "Mirrors, Minds, and Metaphors." Philosophy East and West 58.3: 337-357.

Cserep, Attila. 2014. "Conceptual Metaphor Theory: In Defense of the Fence?" Argumentum 10: 261-288.

Ekstrom, Martin S. 2014. "Does the Metaphor Translate?" In Translating China for Western Readers: Reflective, Critical, and Practical Essays, edited by Gu Ming Dong and Rainer Shulte. Albany: State University of New York Press.

Faucconnier, Gilles. 2003. Mappings in Thought and Language. Cambridge: Cambridge University Press.

Faucconnier, Gilles, and Mark Turner. 2003. The Way We Think: Conceptual Blending and the Mind's Hidden Complexities. New York: Basic Books.

Gibbs, Raymond. 2009. "Why Do Some People Dislike Conceptual Metaphor Theory?" Cognitive Semiotics 5.1: $14-36$

2011. "Evaluating Conceptual Metaphor Theory." Discourse Processes 48.8: 529-562.

2015. "Counting Metaphors: What Does This Reveal about Language and Thought?" Cognitive Semantics 1.2: 155-177.

Giblett, Rod. 2009. "The Tao of Water." Landscapes: The Journal of the International Centre for Landscape and Language 3.2: 15-26.

Glucksberg, Sam. 2008. "How Metaphors Create Categories-Quickly." In Cambridge Handbook of Metaphor and Thought, edited by Raymond W. Gibbs. Cambridge: Cambridge University Press.

Glucksberg, Sam, and Boaz Keysar. 1998. "How Metaphors Work." In Metaphor and Thought, edited by Andrew Ortony. Cambridge: Cambridge University Press. 
Gu, Ming Dong. 1997. "Fu-Bi-Xing: A Metatheory of Poetry-Making." Chinese Literature: Essays, Articles, Reviews (CLEAR) 19: 1-22.

Hansen, Chad. 1985. "Chinese Language, Chinese Philosophy, and 'Truth."” The Journal of Asian Studies 44.3: 491-519.

Harrison, Victoria S. 2015. "Seeing the Dao: Conceptual Metaphors and the Philosophy of Religion." Religious Studies 51: 307-322.

He, Liye 何麗野. 2003. "Fire and Water: The Fundamental Metaphors of Chinese and Western Philosophy 水與 火: 中西哲學的核心隱喻和文化的基本精神.” Shehui kexue 社會科學 6: 86-90.

Heidegger, Martin. 1957. The Principle of Reason [Der Satz vom Grund]. Frankfurt: Klostermann.

Hurst, Cecily. 2000. "The Origin of Language in Chinese Thought." Anthropoetics 6.2: 1-15.

Jones, Nicholaos. 2016. “Correlative Reasoning about Water in Mengzi 6A2.” Dao: A Journal of Comparative Philosophy 15.2: 193-207.

Kallay, Geza. 2014. "Some Philosophical Problems about Metaphor." Argumentum 10: 339-345.

Kao, Karl S. Y. 2000. "Comparative Literature and the Ideology of Metaphor, East and West." Comparative Literature and Language 2.4: 1-9.

Kovecses, Zoltan. 2005. Metaphor in Culture: Universality and Variation. Cambridge: Cambridge University Press.

2013. "Recent Developments in Metaphor Theory: Are the New Views Rival Ones?" In Metaphor and Metonymy Revisited beyond the Contemporary Theory of Metaphor: Recent Developments and Applications, edited by Fransisco Gonzalvez-Garcia, Maria Sandra Pena Cervel, and Lorena Perez Hernández. Philadelphia: John Benjamins.

Lakoff, George. 1998. "The Contemporary Theory of Metaphor." In Metaphor and Thought, edited by Andrew Ortony. Cambridge: Cambridge University Press.

Lakoff, George, and Mark Johnson. 1980. Metaphors We Live By. Chicago: Chicago University Press.

Link, Perry. 2013. An Anatomy of Chinese: Rhythm, Metaphor, Politics. Cambridge, MA: Harvard University Press.

Liu, James J. Y. 1975. Chinese Theories of Literature. Chicago: University of Chicago Press.

Lu, Yanying. 2012. "Water Metaphors in Dao De Jing: A Conceptual Analysis." Open Journal of Modern Linguistics 2.4: 151-158.

Makeham, John. 2012. "Introduction." In Learning to Emulate the Wise: The Genesis of Chinese Philosophy as an Academic Discipline in Twentieth-century China, edited by John Makeham. Hong Kong: The Chinese University Press.

de Mendoza Ibáñez, Francisco José Ruiz, and Lorena Pérez Hernández. 2011. "The Contemporary Theory of Metaphor: Myths, Developmentss and Challenges.” Metaphor and Symbol 26.3: 161-185.

Min, Lin. 2001. Certainty as a Social Metaphor. London: Greenwood Press.

Owen, Stephen. 1992. Reading in Chinese Literary Thought. Cambridge, MA: Council on East Asian Studies, Harvard University.

Rakova, Marina. 2002. "The Philosophy of Embodied Realism: A High Price to Pay?" Cognitive Linguistics 13.3: $215-244$.

Reding, Jean-Paul. 2004. Comparative Essays in Early Greek and Chinese Rational Thinking. Adlershot: Ashgate.

Slingerland, Edward. 2003. Effortless Action: Wu-wei as Conceptual Metaphor and Spiritual Ideal in Early China. Oxford: Oxford University Press.

2004a. "Conceptions of the Self in the Zhuangzi: Conceptual Metaphor Analysis and Comparative Thought." Philosophy East and West 54.3: 322-342.

2004b. "Conceptual Metaphor Theory as Methodology for Comparative Religion." Journal of the American Academy of Religion 72.1: 1-31.

2005. "Conceptual Blending, Somatic Marking, and Normativity: A Case Example from Ancient Chinese." Cognitive Linguistics 16.3: 557-584.

. 2011. "Metaphor and Meaning in Early China." Dao: A Journal of Comparative Philosophy 10.1: 130.

2017. "Metaphor, Blending, and Cultural Variation: A Reply to Camus." Dao: A Journal of Comparative Philosophy 16: 431-435.

Steen, Gerard. 2011. "The Contemporary Theory of Metaphor-Now New and Improved!” Review of Cognitive Linguistics 9.1: 26-64.

Wu, Yameng 吳雅萌. 2011. “Cognitive Difference in the Metaphorical Use of 'Water' between English and Chinese 英漢“水”隱喻的認識分析.” Mudanjiang daxue xuebao 牡丹江大學學報 11: 60-62.

Wu, Zhongsheng 吳中勝. 2016. “Water and Literature in The Literary Mind and the Carving of Dragons 從 “上 善若水’ 到《文心雕龍》的 “以水喻文”.” Zhongzhou xuekan 中州學刊 6: 132-137. 
Yang, Yanning. 2011. "Grammatical Metaphor in Chinese: A Corpus Based Study." Functions of Language 18.1: 1-28.

Yip, Wai-Lim. 1993. Diffusion of Distances: Dialogues between Chinese and Western Poetics. Berkeley: University of California Press.

Yu, Ning. 1998. The Contemporary Theory of Metaphor: A Perspective from Chinese. Philadelphia: John Benjamins.

Yu, Pauline. 1987. The Reading of Imagery in the Chinese Poetic Tradition. Princeton: Princeton University Press.

Yu, Pauline, and Theodore Huters. 1994. “The Imaginative Universe of Chinese Literature.” In Masterworks of Asian Literature in Comparative Perspective: A Guide for Teaching, edited by Barbara Stoler Miller. Arnok: M. E. Sharp.

Zhang, Longxi. 1996. "What Is Wen and Why Is It Made So Terribly Strange?" College Literature 23.1: 1535 .

Zhang, Shaokang 張少康. 1993. New Investigations on The Literary Mind and the Carving of Dragons 文心雕 龍新探. Taipei 台北: Wenshizhe chubanshe 文史哲出版社.

Publisher's Note Springer Nature remains neutral with regard to jurisdictional claims in published maps and institutional affiliations. 